\title{
Endovascular Treatment of a Hepatic Artery Pseudoaneurysm Using a Novel Pericardium Covered Stent
}

\author{
Authors: \\ Brett Larner ${ }^{1}$, Julian Maingard2,3,4, Yifan Ren ${ }^{1}$, Hong Kuan Kok ${ }^{4,5}$, Ronil V. Chandra ${ }^{6,7}$, Michael J \\ Lee $^{8,9}$, Anthony Schelleman², Duncan Mark Brooks ${ }^{2,3,4,10}$, Hamed Asadi2,3,4,6,10
}

Affiliation:

${ }^{1}$ Austin Hospital, Melbourne, Australia.

2 Interventional Radiology Service - Department of Radiology, Austin Hospital, Melbourne, Australia.

${ }^{3}$ Interventional Neuroradiology Service - Department of Radiology, Austin Hospital, Melbourne, Australia.

${ }^{4}$ School of Medicine - Faculty of Health, Deakin University, Waurn Ponds, Australia.

${ }^{5}$ Interventional Radiology Service - Department of Radiology, Northern Hospital, Melbourne, Australia,

${ }^{6}$ Interventional Neuroradiology Unit, Monash Imaging, Melbourne, Australia.

${ }^{7}$ Faculty of Medicine, Nursing and Health Sciences, Monash University, Melbourne, Australia.

${ }^{8}$ Interventional Radiology Service - Department of Radiology, Beaumont Hospital, Dublin, Ireland.

${ }^{9}$ Royal College of Surgeons in Ireland, Dublin, Ireland.

${ }^{10}$ Stroke Division, Florey Institute of Neuroscience and Mental Health, University of Melbourne, Australia.

Address for Correspondence:

Brett Larner (brett.larner@gmail.com)

This is the author manuscript accepted for publication and has undergone full peer review but has not been through the copyediting, typesetting, pagination and proofreading process, which may lead to differences between this version and the Version of Record. Please cite this article as doi: $10.1111 / 1754-9485.12960$

This article is protected by copyright. All rights reserved 
Austin Hospital, 145 Studley Road, Heidelberg, Victoria 3084, Australia.

Mobile: +61400973340

Total Word Count: 2859

Reference count: 21

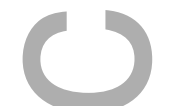

Level of Evidence: Level 4, Technical Report.

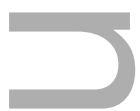

Compliance with Ethical Standards: This study was not supported by any funding. The authors declare that they have no conflict of interest. All procedures performed in studies involving human participants were in accordance with the ethical standards of the institutional and/or national research committee and with the 1964 Helsinki declaration and its later amendments or comparable ethical standards. Informed consent was obtained from all individual participants included in the study. Consent for publication was obtained for every individual person's data included in the study.

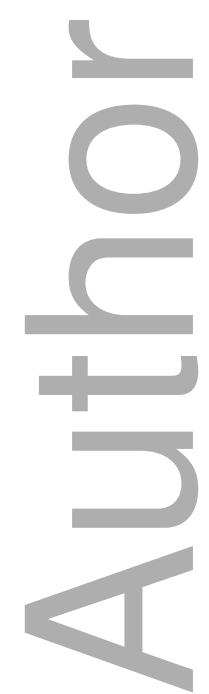

This article is protected by copyright. All rights reserved 
4

5

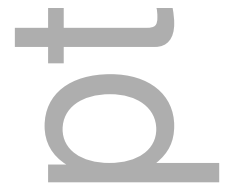

Article type : Radiology Technical Article
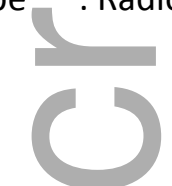

\section{Abstract}

Visceral and renal artery aneurysms (VRAAs) and pseudoaneurysms are rare. Their increasing incidence is largely thought to be due to advances in medical imaging. Twenty percent of VRAAs occur in hepatic arteries, with approximately fifty percent of these represented by pseudoaneurysms, which are prone to spontaneous rupture. Many treatments for VRAAs exist, with the endovascular approach being favoured. Treatment aims to preserve visceral perfusion and exclude the aneurysm; however complex aneurysms may require parent artery or end-organ sacrifice. Covered stents allow rapid aneurysm exclusion while preserving parent artery patency, a favourable outcome when parent artery or end-organ sacrifice is undesirable. The AneuGraft pericardium covered stent (PCS) combines the benefits of a low-profile covered stent with those of a low immunogenic material. We describe the endovascular treatment of a patient with a hepatic artery pseudoaneurysm, where parent artery sacrifice was considered unacceptable. The AneuGraft PCS was used to provide immediate and complete exclusion, with dual antiplatelet therapy for 1 week, followed by single antiplatelet use. The procedure was a technical success, with preservation of the hepatic arteries, and complete exclusion of the pseudoaneurysm. There were no complications immediately following the procedure, or on post-procedural follow up. The pseudoaneurysm remained excluded at 6-week CTA follow up. This case describes a safe and effective method for completely excluding a complex pseudoaneurysm, utilising the AneuGraft PCS, allowing for the potential management of a wider range of aneurysms with unfavourable morphology.

\section{Background}

Visceral and renal artery aneurysms (VRAAs) and pseudoaneurysms occur rarely, with a reported prevalence between $0.1-2 \%(1-3)$. Increases in the number of percutaneous biliary tract procedures and laparoscopic treatments, and advances in medical imaging are thought to contribute to the increasing incidence of VRAA and pseudoaneurysm diagnoses (2-4). Twenty percent of all VRAAs 
occur in branches of the hepatic arteries, with approximately fifty percent of these represented by pseudoaneurysms $(1,5,6)$. Pseudoaneurysms are prone to spontaneous rupture into both the biliary

3 tree and peritoneum, resulting in significant morbidity and mortality (1, 3-5, 7). Therefore, visceral 4 pseudoaneurysms of any size at any location require prompt treatment $(3,4,7,8)$.

5 There are a wide variety of endovascular approaches described for VRAA treatment but the common aim is to exclude the aneurysm and preserve parent vessel patency and visceral perfusion, where possible (3). Parent arteries with a tortuous path, wide-necked aneurysms, and aneurysms located at arterial bifurcations present challenges to aneurysm occlusion (9). Such aneurysms typically require parent artery sacrifice or adequate exclusion to prevent complications or recurrence of the aneurysm. However, the former requires intact collateral blood supply of the viscera to preserve end-organ perfusion $(3,9,10)$. This is of particular importance for transplanted viscera in which collateral blood supply may be absent, resulting in visceral ischaemia or infarction (11).

Covered stent grafts permit complete exclusion of an aneurysm or pseudoaneurysm while maintaining parent artery patency, overcoming the ischaemic risk associated with parent artery sacrifice and embolisation of the aneurysm neck (12). Synthetic stent coverings such as PTFE have been shown to promote thrombosis and slow endothelialisation $(10,13)$. Pericardium is a well utilised graft material, suitable for use as a stent covering with low immunogenicity and high durability $(13,14)$. In our experience early institution of dual antiplatelet therapy using $100 \mathrm{mg}$ aspirin and $75 \mathrm{mg}$ clopidogrel is used for many cases, however the pericardium covering allows for single antiplatelet therapy when dual antiplatelet therapy is not indicated or is inappropriate.

The AneuGraft pericardium covered stent (PCS) (Amnis Therapeutics Ltd, Or Akiva, Israel) (Fig. 1) is a highly flexible, laser cut, $316 \mathrm{~L}$ stainless-steel balloon-expandable stent, which is covered with a single layer of equine pericardium delivered through a 6 French guiding catheter, trackable over a 0.014" guidewire allowing for deployment in smaller and more distal vessels. It is designed for use in vessels from $2.5 \mathrm{~mm}$ to $4.0 \mathrm{~mm}$ in diameter and comes in a range of lengths from $13 \mathrm{~mm}$ to $27 \mathrm{~mm}$. The AneuGraft PCS, with its low profile and high flexibility, has been shown to have increased deliverability when compared to the Graftmaster PTFE-covered stent (Abbott Vascular, Santa Rosa, $\mathrm{CA}$ ) in both phantom and porcine coronary artery models (15).

The AneuGraft PCS has been used successfully in the management of coronary stenosis and coronary aneurysms $(16,17)$. There are few studies that have examined the AneuGraft PCS in the management of non-cardiac aneurysms. Case studies outlining its use in the management of hepatic artery pseudoaneurysms and internal carotid and vertebral artery aneurysms have reported complete aneurysm exclusion and maintenance of parent artery patency for all patients $(8,13,18)$. Currently, the AneuGraft PCS is TGA approved for use in the coronary circulation. Here we describe the offlabel use of the AneuGraft PCS to treat a pseudoaneurysm of the right hepatic artery in a post-liver transplant patient. 


\section{Case Report}

2

3

\section{Presentation}

A 58 year-old-male presented 7 months after an orthoptic liver transplant for alcoholic cirrhosis with nausea, vomiting, and generalised abdominal pain. A CT abdomen showed a periportal collection, small bowel obstruction, and a proximal right hepatic artery pseudoaneurysm (Fig. 2A), which required prompt treatment. The patient had a percutaneous pigtail catheter inserted to drain the periportal collection, and a biliary stent for a post-transplant ischaemic stricture. The presence of an occlusive portal vein thrombus at the anastomosis made open surgical and endovascular approaches of parent artery sacrifice less favourable in view of the compromised portal supply, where preservation of hepatic artery patency was preferred to prevent visceral ischaemia. As such, an endovascular strategy utilising a covered stent graft was chosen to exclude the pseudoaneurysm over other available flow modulating devices (such as flow diverting stents). Given the patient was acutely unwell and there was a possibility of needing to return to theatre for either a washout or repeat liver transplant, single antiplatelet therapy was determined to be more appropriate.

\section{Procedure}

Following written informed consent, under sterile technique and general anaesthesia, the right common femoral artery (CFA) was visualised under ultrasound guidance and punctured, before insertion of an 8-French introducer sheath (Terumo, Tokyo, Japan). After 5000 units of intra-arterial heparin and $500 \mathrm{mg}$ IV Aspirin, the coeliac trunk, transplanted common hepatic artery, and right hepatic artery were selectively catheterised with a SIM 2 catheter (Cook Medical, Bloomington, USA). Digital subtraction angiography (DSA) confirmed a $2.7 \mathrm{~cm}$ pseudoaneurysm in the proximal right hepatic artery (Fig. 2B). The SIM 2 catheter was subsequently exchanged for a guide catheter (Neuron MAX 088, MicroVention, California, USA) over an exchange length wire. 5-French and 6French intermediate catheters (Sofia, MicroVention, California, USA) together with a microcatheter (SL10, Stryker, Fremont CA, USA) were used to define the in and out-flow anatomy of the pseudoaneurysm and achieve a stable distal position for device delivery (Fig. $2 \mathrm{C}$ ). The 6-French intermediate catheter was used in the event that more microcatheters were required to navigate the pseudoaneurysm, with the 5-French intermediate catheter being used due to its longer length. Following definition of the pseudoaneurysm, a $2.5 \times 18 \mathrm{~mm}$ AneuGraft PCS (Amnis Therapeutics Ltd, OR, Akiva, Israel) was deployed across the neck of the pseudoaneurysm over a synchro soft guidewire (Stryker, MI, USA), preserving the left hepatic artery branch (Fig. 2D). The stent was balloon dilated to $3 \mathrm{~mm}$ and $2.5 \mathrm{mg}$ intra-arterial tirofiban was used to treat a small focus of developing platelet aggregation (Fig. 2E). After stent deployment, final DSA showed complete exclusion of the pseudoaneurysm with maintained distal perfusion (Fig. 2F). Haemostasis was achieved with an 8French vascular closure device (AngioSeal, Terumo, Tokyo, Japan). The patient was continued on a short course of dual antiplatelet therapy for 1 week and subsequently continued on $100 \mathrm{mg}$ aspirin daily indefinitely. 


\section{Results}

3 The procedure was a technical success, with preservation of right and left hepatic artery perfusion,

4 and full exclusion of the aneurysm. There were no immediate complications following the procedure,

5 nor during the post-procedural follow up. Multiphase CT angiography performed 6-week post-

6 procedure showed complete exclusion of the pseudoaneurysm (Fig. 2G). Both right and left hepatic

7 arteries were preserved. No ischaemic complications were encountered.

8

\section{Discussion}

The frequency of asymptomatic VRAA and pseudoaneurysm diagnoses has increased with advancements in the use of cross-sectional imaging (3). Although treatment guidelines vary for true aneurysms, intervention is suggested for pseudoaneurysms at any location and of any size, due to high morbidity and mortality of $70 \%$ associated with rupture $(4,7,8)$. Traditionally VRAA and pseudoaneurysm treatment has involved open surgical approaches, including aneurysm ligation, aneurysm resection and subsequent parent artery reunion, and resection of the end-organ, but is associated with a significant morbidity in up to $18 \%$ of patients $(2,6)$. In comparison, endovascular approaches to treatment are now considered first line, associated with $3.7 \%$ morbidity, $1.5 \%$ mortality at 30 days, and $4.4 \%$ reintervention rates with a $93.6 \%$ technical success rate and preserved visceral perfusion in $99.1 \%$ (10). Endovascular treatment algorithms involve exclusion of the aneurysm with flow modulation (flow diverting or covered stents), or embolisation of the aneurysm via coils or liquid embolic agents (3). Treatment of complex aneurysms and pseudoaneurysms may require alternative methods such as parent artery sacrifice or parent artery remodelling (9).

Covered stents are one of many approaches available to manage aneurysms. Such stent treatments have shown to provide complete exclusion of an aneurysm while maintaining parent artery patency, allowing for the successful treatment of complicated aneurysms and avoiding the need for end-organ resection, particularly useful when treating high risk pseudoaneurysms, although ongoing surveillance of stent position and patency may be warranted $(8,18)$. The AneuGraft PCS has been used extensively in Cardiology practice and is currently indicated for the management of both coronary bypass-vein graft stenosis and coronary bypass-vein graft aneurysms (14). There is limited data directly comparing the AneuGraft PCS to other covered stents, with one study reporting the rates of thrombosis in PTFE-covered stents and pericardium covered stents as high as $8.6 \%$ and $5.7 \%$, respectively. They also showed no significant difference in rates of in-stent restenosis (ISR) (19). However, other studies have independently shown ISR rates as high as $54.6 \%$ for PTFE-covered stents (20), and $26.3 \%$ for PCS (21). Little evidence exists for its use for other indications, however it has been reported as safe in the management of coronary vessel rupture and exclusion of coronary aneurysms $(16,17,21)$. There are few case reports that explore the use of the AneuGraft PCS 
beyond the coronary circulation. Corti et al. reported complete exclusion of a hepatic artery pseudoaneurysm and preservation of a ruptured renal artery, in parent arteries that were not considered to be expendable. They showed that at six months, vessel patency was at $65 \%$, and there were no signs or symptoms related to vessel occlusion or end-organ dysfunction (18). Treatment of internal carotid artery and vertebral artery aneurysms was achieved by Vulev et al (13). The AneuGraft PCS was used in these cases to reduce the risk of complications such as aneurysm rerupture, and in-stent stenosis and thrombosis associated with endovascular coiling and flow diverting stents. Deployment of the stent over the neck allowed for complete exclusion, with one aneurysm requiring two stents to bridge the whole neck. Three month follow-up CTA identified complete exclusion and shrinkage of the aneurysm (13). Ferlini et al. illustrated the safe use of the AneuGraft PCS in the management of a large iatrogenic pseudoaneurysm of the hepatic artery. Complete exclusion of the pseudoaneurysm with no procedure related complications was noted at one week follow up (8). Beyond this, the stent has the potential for occlusion of vessel side branches, which must be taken into account by the operator (13). Here we used the pericardium covered stent to allow for effective exclusion of the pseudoaneurysm while reducing the risk of complications such as instent restenosis and thrombus formation, associated with the use of bare metal stents and PTFEcovered stents $(19,21)$. Given the lack of a true aneurysm wall, coil embolisation was avoided to reduce the risk of pseudoaneurysm expansion or rupture and haemorrhage (13). Because of increased graft porosity and metal coverage ranging from $20-50 \%$, the use of flow diverting stents is not generally accepted as an efficacious method to treat pseudoaneurysms (7), and as such was avoided here. Furthermore, the AneuGraft PCS provided a suitable alternative to bare metal and PTFE-covered stents given the preference for single antiplatelet use in this patient.

This case describes the difficulties of treating an aneurysm in which parent artery patency and endorgan perfusion are to be maintained. The pseudoaneurysm described above was at a high risk of rupture given its size, and was further complicated by its wide-necked morphology and a concomitant occlusive portal vein thrombus. As the patient had received a liver transplant 7 months earlier and the occlusive portal vein thrombus was present, preservation of hepatic artery flow was of utmost importance to obviate ischaemic complications within the graft. It was unknown whether collateral vessels capable of adequately supplying the liver had formed in this patient. As such, there was significant risk of liver ischaemia in the event of pseudoaneurysm rupture or occlusion of the parent artery via endovascular procedures or thromboembolism. The tendency of visceral pseudoaneurysms to rupture and the significant morbidity and mortality associated with such an event, coupled with the inherent complexities of many aneurysms, drives the need for the development and utilisation of safer and more effective devices.

\section{Conclusion}

The AneuGraft PCS has demonstrated early safety and efficacy for complete exclusion of a widenecked hepatic artery pseudoaneurysm. This case describes another safe and effective method for 


\section{Acknowledgements}

5

6

\section{$7 \quad$ References}

8 1. Panayiotopoulos YP, Assadourian R, Taylor PR. Aneurysms of the visceral and renal arteries.

$9 \quad$ Ann R Coll Surg Engl. 1996;78(5):412-9.

completely excluding complex aneurysms from the circulation, allowing for the potential management of a wider range of complex aneurysms with unfavourable morphology.

Not applicable. 2. Tulsyan N, Kashyap VS, Greenberg RK, Sarac TP, Clair DG, Pierce G, et al. The endovascular management of visceral artery aneurysms and pseudoaneurysms. Journal of Vascular Surgery. 2007;45(2):276-83.

3. Belli AM, Markose G, Morgan R. The Role of Interventional Radiology in the Management of Abdominal Visceral Artery Aneurysms. Cardiovascular and Interventional Radiology. 2012;35(2):23443.

4. Reiter DA, Fischman AM, Shy BD. HEPATIC ARTERY PSEUDOANEURYSM RUPTURE: A CASE REPORT AND REVIEW OF THE LITERATURE. Journal of Emergency Medicine. 2013;44(1):100-3. 5. Jeans PL. HEPATIC ARTERY ANEURYSMS AND BILIARY SURGERY TWO CASES AND A LITERATURE REVIEW. Australian and New Zealand Journal of Surgery. 1988;58(11):889-94.

6. Abbas MA, Fowl RJ, Stone WM, Panneton JM, Oldenburg WA, Bower TC, et al. Hepatic artery aneurysm: Factors that predict complications. Journal of Vascular Surgery. 2003;38(1):41-5.

7. Loffroy R, Favelier S, Pottecher P, Genson PY, Estivalet L, Gehin S, et al. Endovascular management of visceral artery aneurysms: When to watch, when to intervene? World Journal of Radiology. 2015;7(7):143-8.

8. Ferlini M, Quaretti P, Cascella T, Cionfoli N, Ferrario M, Bramucci E. Percutaneous hepatic pseudoaneurysm exclusion using a pericardium covered stent. Journal of Cardiovascular Medicine. 2013;14(6):472-3.

9. Maingard J, Kok HK, Phelan E, Logan C, Ranatunga D, Brooks DM, et al. Endovascular Treatment of Wide-Necked Visceral Artery Aneurysms Using the Neurovascular Comaneci NeckBridging Device: A Technical Report. Cardiovasc Intervent Radiol. 2017;40(11):1784-91.

10. Kok HK, Asadi H, Sheehan M, Given MF, Lee MJ. Systematic Review and Single-Center Experience for Endovascular Management of Visceral and Renal Artery Aneurysms. Journal of Vascular and Interventional Radiology. 2016;27(11):1630-41. 
11. Tzakis AG, Gordon RD, Shaw BW, Iwatsuki S, StarzI TE. CLINICAL PRESENTATION OF HEPATICARTERY THROMBOSIS AFTER LIVER-TRANSPLANTATION IN THE CYCLOSPORINE ERA. Transplantation. 1985;40(6):667-71.

12. Ikeda O, Nakasone Y, Tamura Y, Yamashita Y. Endovascular Management of Visceral Artery Pseudoaneurysms: Transcatheter Coil Embolization Using the Isolation Technique. Cardiovascular and Interventional Radiology. 2010;33(6):1128-34.

13. Vulev I, Klepanec A, Bazik R, Balazs T, Illes R, Steno J. Endovascular Treatment of Internal Carotid and Vertebral Artery Aneurysms Using a Novel Pericardium Covered Stent. Interventional Neuroradiology. 2012;18(2):164-71.

14. Colombo A, Almagor Y, Gaspar J, Vonderwalde C. The pericardium covered stent (PCS). Eurointervention. 2009;5(3):394-9.

15. Jokhi PP, McKenzie DB, O'Kane P. Use of a novel pericardial covered stent to seal an iatrogenic coronary perforation. The Journal of invasive cardiology. 2009;21(10):E187-90.

16. Siregar S, Guyomi SH, van Herwerden LA. Covered stents in giant coronary artery aneurysm. European Heart Journal. 2010;31(22):2823-.

17. Ferlini M, Russo F, Marinoni B, Repetto A, Canosi U, Ferrario M, et al. Percutaneous Coronary Aneurysm Obliteration Using a Novel Pericardium-Covered Stent. Journal of the American College of Cardiology. 2010;56(25):2139-.

18. Corti R, Moramarco LP, Leati G, Fiorina I, Quaretti P. Cardiological pericardium covered stent to treat extracardiac hemorrhagic emergencies. European Congress of Radiology; Vienna, Austria2016.

19. Rosseel L, Scott B, Prihadi E, Azzano A, Degrauwe S, Verheye S, et al. Is a covered stent justifiable in the treatment of coronary artery perforation? An observational analysis of long-term results of two different covered stent types. Catheter Cardiovasc Interv. 2019;93(3):419-25.

20. Copeland KA, Hopkins JT, Weintraub WS, Rahman E. Long-term follow-up of polytetrafluoroethylene-covered stents implanted during percutaneous coronary intervention for management of acute coronary perforation. Catheterization and cardiovascular interventions : official journal of the Society for Cardiac Angiography \& Interventions. 2012;80(1):53-7.

21. Secco GG, Serdoz R, Kilic ID, Caiazzo G, Mattesini A, Parisi R, et al. Indications and immediate and long-term results of a novel pericardium covered stent graft: Consecutive 5 year single center experience. Catheterization and Cardiovascular Interventions. 2016;87(4):712-9. 

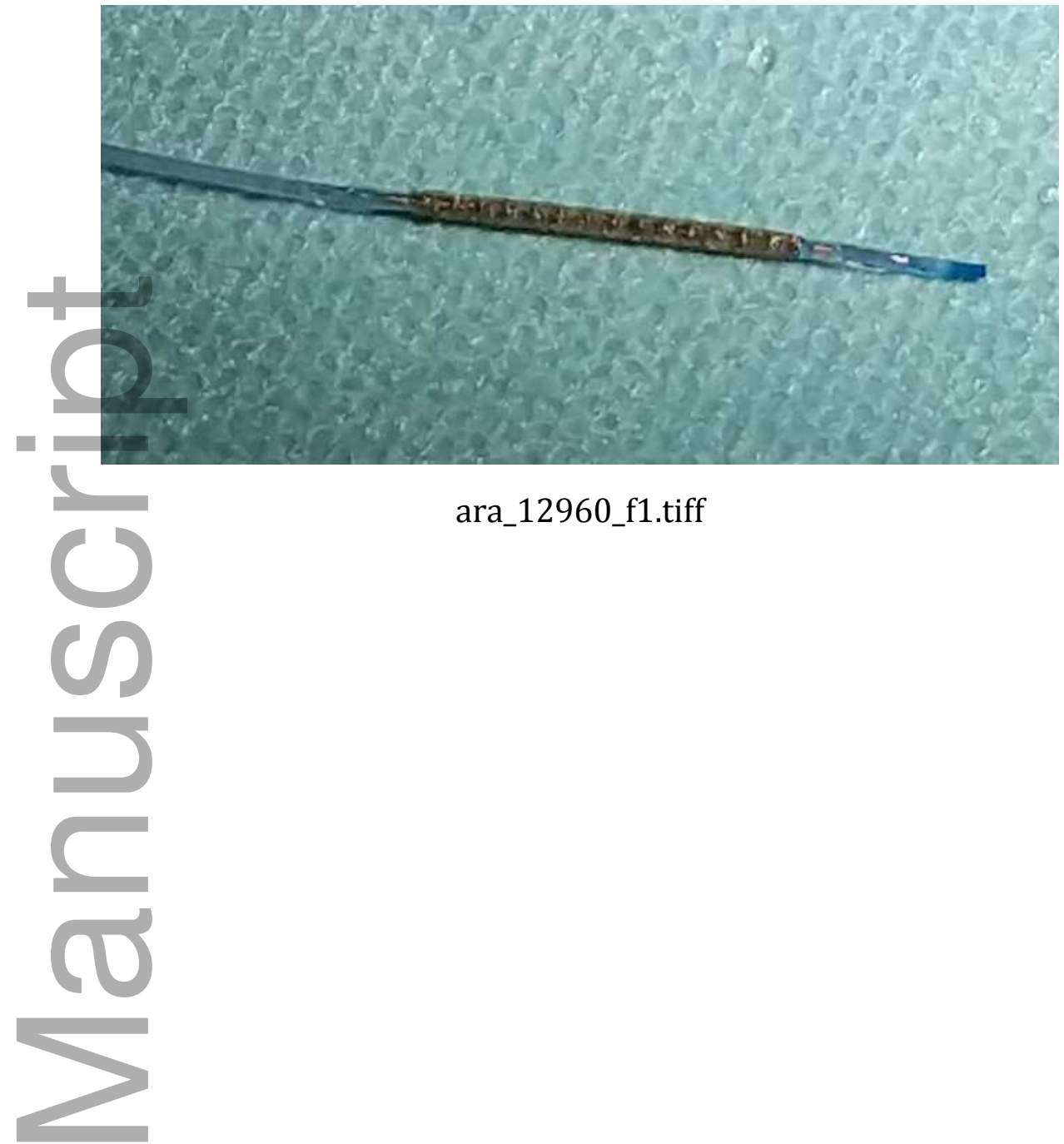

ara_12960_f1.tiff

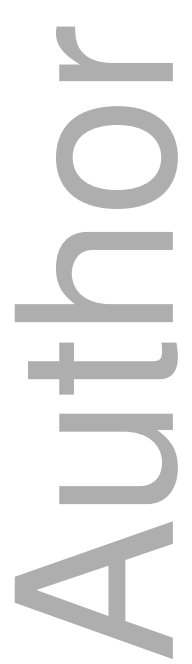

This article is protected by copyright. All rights reserved 

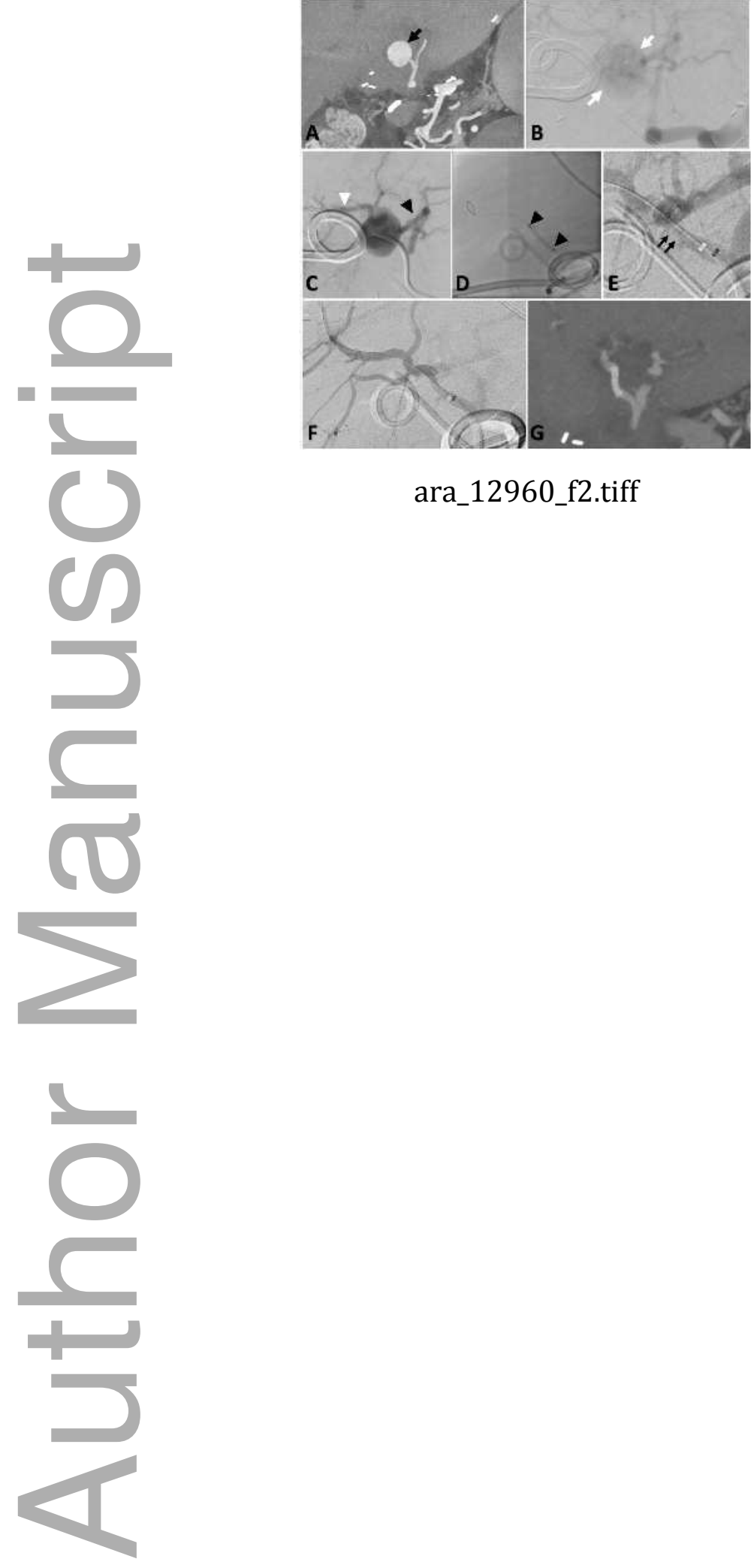

ara_12960_f2.tiff 


\section{University Library}

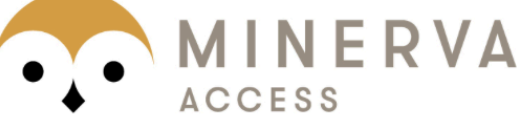

A gateway to Melbourne's research publications

Minerva Access is the Institutional Repository of The University of Melbourne

\section{Author/s:}

Larner, B;Maingard, J;Ren, Y;Kok, HK;Chandra, RV;Lee, MJ;Schelleman, A;Brooks,

DM;Asadi, $\mathrm{H}$

Title:

Endovascular treatment of a hepatic artery pseudoaneurysm using a novel pericardium covered stent

Date:

2019-10-13

Citation:

Larner, B., Maingard, J., Ren, Y., Kok, H. K., Chandra, R. V., Lee, M. J., Schelleman, A., Brooks, D. M. \& Asadi, H. (2019). Endovascular treatment of a hepatic artery pseudoaneurysm using a novel pericardium covered stent. JOURNAL OF MEDICAL IMAGING AND RADIATION ONCOLOGY, 63 (6), pp.765-769. https:// doi.org/10.1111/1754-9485.12960.

Persistent Link:

http://hdl.handle.net/11343/286495 